\title{
Multidisciplinary Approach in a 12-Year-Old Patient Affected by Severe Obstructive Sleep Apnea: a Case-Report
}

\author{
Denis Bignotti, DDS, Alberto De Stefani, DDS, Luca Mezzofranco, DDS, Giovanni Bruno, DDS, Antonio Gracco, DDS
}

Department of Neuroscience, School of Dentistry, University of Padua, Padua, Italy

Received: November 4, 2019

Revised: November 22, 2019

Accepted: November 27, 2019

Correspondence

Alberto De Stefani, DDS

Department of Neuroscience,

School of Dentistry, University of Padua,

Via Giustiniani 2, Padua 35136, Italy

Tel +393343345840

E-mail alberto.de.stefani@hotmail.it

ORCID

Alberto De Stefani

https://orcid.org/0000-0001-9087-3953
A 12-year-old boy shows a restless sleep and snoring episodes declaring himself tired during the day and the school lessons. On clinical examination the patient presents a second-class profile, a retrusive jaw with a small chin and an open nasolabial angle. From the intraoral examination a tonsillar hypertrophy is denoted. The patient is sent by the ear, nose, and throat specialist (ENT) and subsequently subjected to a polysomnography (PSG). The ENT decides to subject him to a tonsillectomy and the subsequent PSG shows an improvement in the obstructive sleep apnea syndrome (OSAS) pathology with an apnea-hypopnea index (AHI) improved from 25.5 episodes/hr to 3.4 episodes/hr. Subsequently a orthodontic treatment with twin-block was start to further reduce the apnea episodes. The last PSG highlights the success of the treatment further lowering the AHI to 0.7 episodes/hr. This case report shows how a multidisciplinary approach to OSAS is fundamental also in the young patient and that the orthodontist carried out in this area a fundamental task both in diagnosis and treatment.

Sleep Med Res 2019;10(2):103-107

\section{INTRODUCTION}

Obstructive sleep apnea syndrome (OSAS) is a breathing sleep disorder that affects $1 \%$ to $3 \%$ of children characterized by repeated episodes of complete and/or partial and/or prolonged upper airway obstruction during sleep, normally associated with a reduction in blood oxygen saturation. Completed obstruction defines apnea episodes while partial obstruction defines hypopnea episodes [1-3].

It is possible to divide the signs and symptoms of OSAS into two groups: the first consists of those directed to the intermittent pharyngeal airway obstruction as the parents report on episodes of apneas or snoring of the child; the second from the comorbidities that develop due to obstruction: daytime sleepiness, hyperactivity, enuresis, inadequate body growth, and poor academic results [4].

In addition, several studies show the great medical implications caused by OSA: metabolic, endocrine, and cardiovascular problems that undermine the proper development of the young individual [5].

The gold standard for evaluation of pediatric OSAS is polysomnography (PSG) and the apnea-hypopnea index (AHI) is the most commonly used PSG parameter for the quantification of sleep disorders breathing severity. It includes the number of mixed, obstructive, and central apneas and hypopneas for hours of total sleep time [6].

In childhood an obstructive $\mathrm{AHI} \leq 1$ episodes/hr is considered to be normal, $1<\mathrm{AHI} \leq 5$ to be mild OSA, $5<\mathrm{AHI} \leq 10$ to be moderate OSA, and an AHI $>10$ episodes/hr as severe OSA [7].

The surgical treatment of first choice in children with OSAS is adenotonsillectomy. The first multisite, randomized controlled, and prospective study investigating the effects of adenoton- 
sillectomy on OSAS treatment confirmed that the surgical option, compared to the wait-and-see option, had improved behavior and quality of life in the short and long term and also given better results obtained with PSG in resolving OSA [8]. To the same result comes the meta-analysis of Brietzke and Gallagher [6] stating that adenotonsillectomy is a successful way to normalize PSG measurements in the majority of patients. The authors add, however, that given the complexity of the OSA problem, the presence of residual apnea episodes must push the research to investigate the pathology and the medical team to resolve the problem from more therapeutic fronts.

Regarding non-surgical treatments, continuous positive airway pressure (CPAP), that is the most common treatment in adult patients, seemed not ideal for children due to a very poor compliance rate [9].

From these assumptions orthodontic treatment became essential which in children mainly involves two alternatives: the palatal expansion in those patients with narrow palates and mandibular advancement with dedicated devices, also chosen taking into account the skeletal typology and growth who is facing the young patient [10].

\section{CASE REPORT}

A 12-year-old boy presented at the Padua University Hospital Dental Clinic for an orthodontic evaluation. The general medical history does not present any known pathology but the parents suspect that the child has apnee during the night and they also communicate snoring episodes. The patient declares himself tired during the day and shows a difficulty in handling attention during school lessons. At the first visit he presents a body mass index (BMI) of 22.2 and a result for the assessment of daytime sleepiness (Epworth test) of 4 that is a seemingly normal value. At the frontal extraoral evaluation, there is a reasonable proportion of the thirds with a slight mandibular deviation towards the right. In the sagittal vision it presents a second-class profile, a retrusive jaw with a little chin and an open nasolabial angle.

From the intra-oral exam it presents a second molar class of Angle on the right and a first on the left and a second canine class. The midline lines are centered but the upper right lateral incisor is small. To conclude the upper and lower crowding is absent. At the objective examination, a tonsil hypertrophy is also noted. In order to analyze more extensively her condition an ear, nose, and throat specialist evaluation was required. A cone beam computed tomography of the craniofacial complex was requested and the patient was referred by an otolaryngologist for a specialist examination (Figs. 1 and 2).

The specialist confirms the hypertrophy and first of all proposes a PSG a to monitor the patient's sleep. The instrumental framework shows respiratory events of a mainly obstructive nature, expressed mostly during supine decubitus, with a severe degree of apnea hypopnea. Specifically, the test reports an AHI of $25.5 / \mathrm{h}$, which doubled the cut-off for the diagnosis of severe OSA, the mean minimum desaturation values is $93.5 \%$, the average saturation in sleep is $97.3 \%$, the nadir of $89 \%$, the average night heart rate is $65.0 \mathrm{bpm}$. 95\% of apnea events were obstructive, the remaining $5 \%$ were a combination of obstructive and central events.

The cause of these results is found in tonsillar hypertrophy and

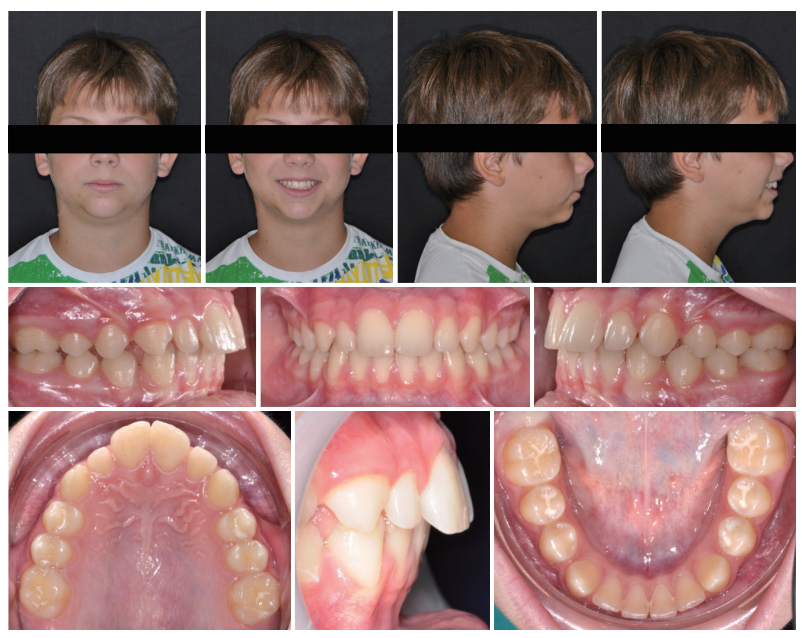

Fig. 1. Initial records: intraoral and extraoral photographs.
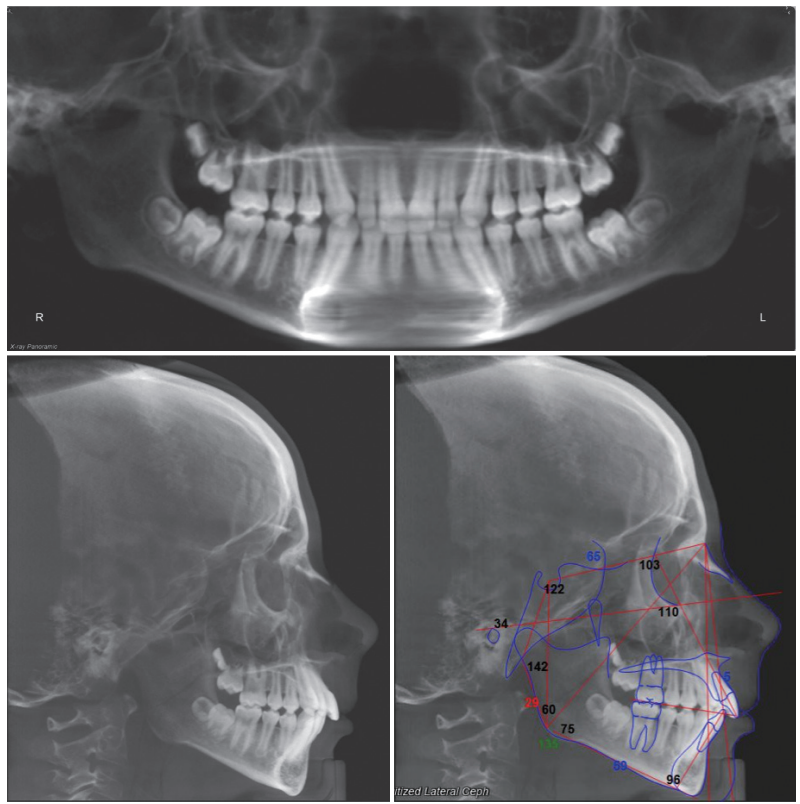

Fig. 2. Initial x-rays: orthopanoramic and lateral radiographs and cephalometric analysis.

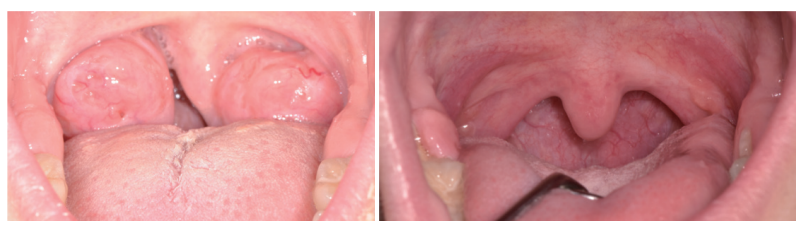

Fig. 3. Tonsillectomy. Pre-operation and post-operation. 
we proceed with tonsillectomy (Fig. 3, is visible the pre-operation and the post-operation image of the tonsils). After the surgical operation and the patient's recovery, a second PSG is performed and the following results are detected: AHI score of $3.4 / \mathrm{h}$, average oxygen saturation of $96 \%$, nadir of $93 \%$, oxygen desaturation index (ODI) of 0.7 , and average night heart rate equal to $54.0 \mathrm{bpm}$ (Table 1). The surgical operation has therefore significantly improved the pathological picture of the young patient but it was not enough to bring the AHI below 1 and thus fully resolve the OSA pathology. At this point orthodontic therapy comes into play. As is known in the literature, CPAP is the most effective treatment but it has a very poor compliance rate. Furthermore, CPAP therapy can also affect the physiological skeletal development of craniofacial structures in a growing patient. Therefore, it was decided to opt for a mandibular advancement device (MAD) that would also meet its skeletal typology and that would also encourage the retruded jaw to express its maximum growth potential. Rapid maxillary expansion was excluded as a possible treatment because authors did not evidence a maxillary deficiency in this patient. Since the BMI of the patient (22.2) was in a normal range for his age, weight loss was excluded too.

\section{The Orthodontic Phase}

When the diagnostic records are updated, the patient's occlusal and facial features remain almost the same (Fig. 4).

The treatment consists of a first step of taking precision impressions with polyvinylsiloxane taken with George Gauge in 0 $\mathrm{mm}$ overjet relation. With this material the MAD is constructed which, in the specific case of this patient, is a twin block.

Delivery of the twin block with indication to the patient to bring the device in the afternoon and at night to counteract the apneas during the night and possibly stimulate the jaw to grow. The patient is seen about every month and a half for control and to report the patient's impressions. The patient immediately dem-

Table 1. Results of the three polysomnographs

\begin{tabular}{lccc}
\hline & PSG pre & $\begin{array}{c}\text { PSG post } \\
\text { tonsillectomy }\end{array}$ & $\begin{array}{c}\text { PSG post } \\
\text { twin block }\end{array}$ \\
\hline Total recording time (min) & 452 & 420 & 360 \\
Snoring time (min) & 0.1 & 24.0 & 0.2 \\
Time in the supine position (\%) & 17.8 & 35.6 & 28.1 \\
Apnea-hypopnea index (h) & 25.5 & 3.4 & 0.7 \\
Apnea-hypopnea index & 90.5 & 0.8 & 0 \\
supine (h) & & & 96.0 \\
Average saturation (\%) & 97.3 & 96.0 & 50 \\
Nadir (\%) & 89 & 93 & 3.7 \\
Oxigen desaturation index & 22.0 & 0.7 & \\
Time of sleep with saturation & 0.1 & 0 & \\
under 90\%, CT90 (\%) & & &
\end{tabular}

PSG: polysomnography. onstrates excellent compliance and declares himself satisfied with the use of the device, feeling even more rested during the day.

After one year of therapy, it is decided to re-evaluate the patient and limit the use of the device only at night. Clinical examination shows that the facial features are stable with a second skeletal class and maxillary bi-retrusion. The intraoral examination shows how a first molar class is obtained and a first canine class on the left. On the right is still a slight second canine class. The overjet relation is close to $0 \mathrm{~mm}$ (Fig. 5) but as expected in the following months with the use of the device only at night, there was a recurrence which led to a more physiological occlusion (Fig. 6).

At this point we decide to perform a third PSG with the MAD in the mouth to understand what are and are present to the patient. The results of this last test indicate an $\mathrm{AHI}$ of $0.7 / \mathrm{h}$ with a total number of apneas equal to 0 and a total of hypopneas of 2 . The average saturation of oxygen is $96 \%$ a nadir of 50 and an

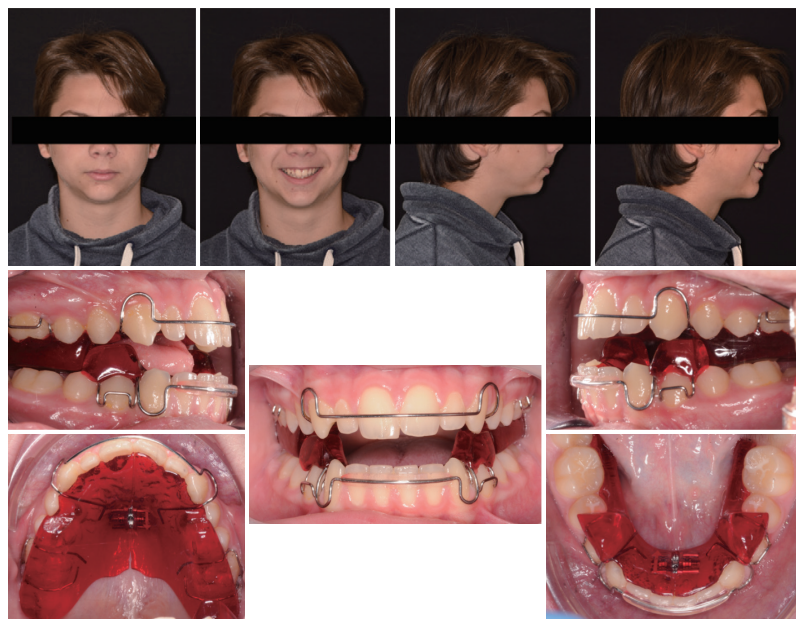

Fig. 4. Records with patience wearing the twin block.
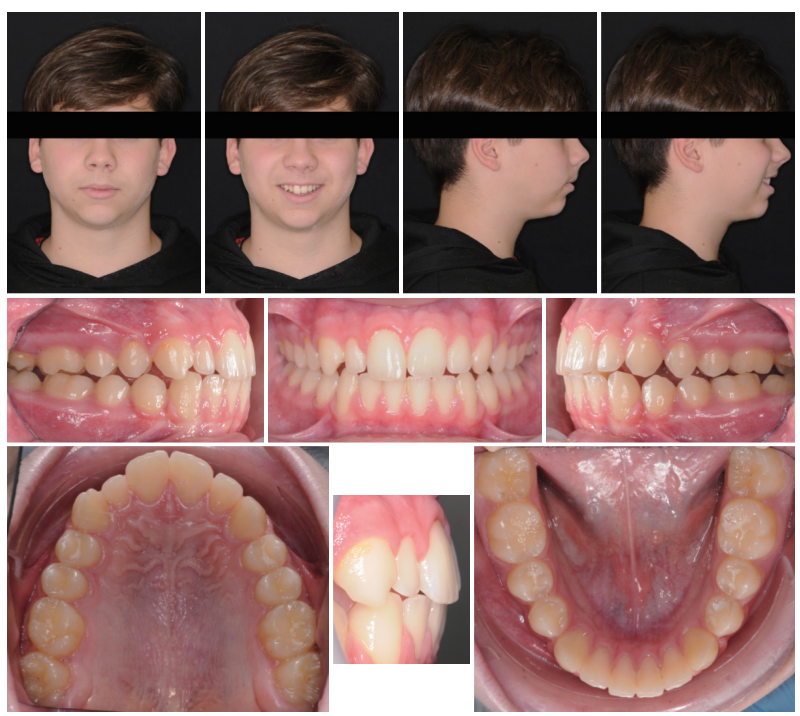

Fig. 5. Records after 1 year of therapy and before the third polysomnography. 
ODI of 3.2 (Table 1 ).

\section{DISCUSSION}

Intervention in the treatment of OSAS in pediatric patients is fundamental for the correct psycho-physical development of the patient. The effectiveness of tonsillectomy in OSA treatment has been investigated in the literature as demonstrated in the introduction and was very effective even with our patient. In fact, the AHI has dropped from 25.5 episodes per hour to 3.4 episodes per hour. This result, although satisfactory, is still not sufficient to consider the OSA completely resolved in a young patient. Generally speaking, orthodontically the first aspect to

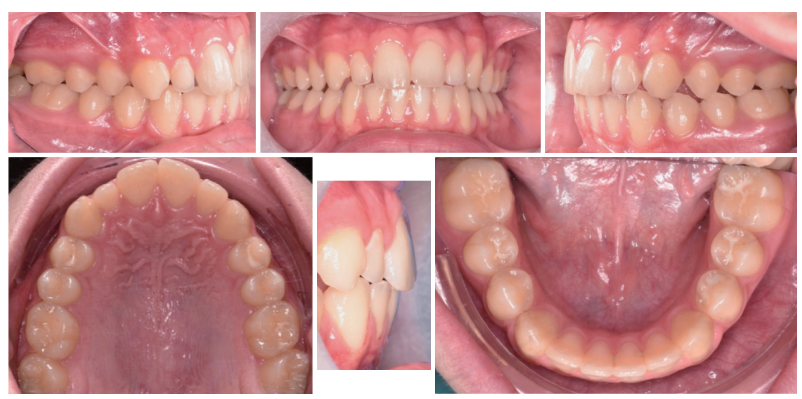

Fig. 6. Intraoral photographs after treatment.

Table 2. Pre and post cephalometric values

\begin{tabular}{lrr}
\hline & Initial & Final \\
\hline Articular angle $\left(^{\circ}\right)$ & 141.8 & 148.9 \\
Gonial angle $\left(^{\circ}\right)$ & 134.6 & 125.0 \\
Anterior cranial base $(\mathrm{mm})$ & 64.8 & 68.1 \\
Ramus height $(\mathrm{mm})$ & 29.4 & 38.7 \\
Corpus lenght $(\mathrm{mm})$ & 59.4 & 62.3 \\
P-A face height $(\%)$ & 59.2 & 59.7 \\
ANB angle $\left(^{\circ}\right)$ & 5.3 & 5.9 \\
IMPA angle $\left(^{\circ}\right)$ & 96.2 & 107.5 \\
$\mathrm{U} 1-\mathrm{FH}\left(^{\circ}\right)$ & 109.7 & 107.1 \\
\hline
\end{tabular}

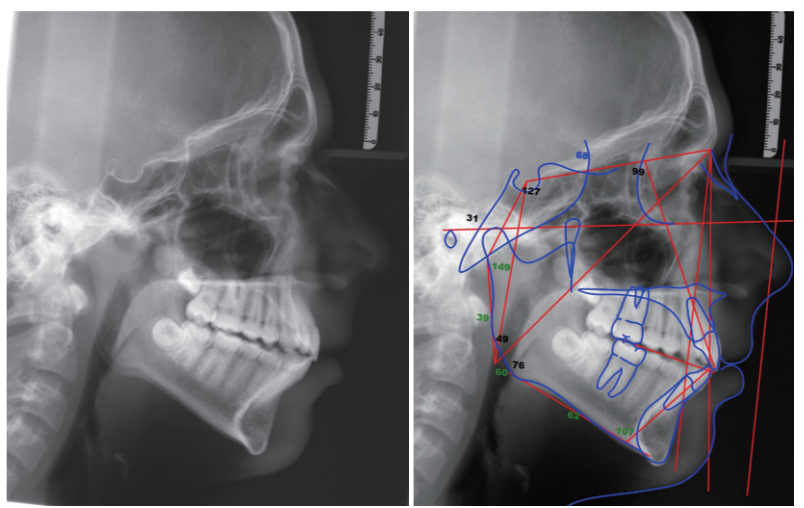

Fig. 7. X-rays after 1 year of therapy and before the third polysomnography. be solved in a pediatric patient with apneas is maxillary constriction; indeed, many studies confirm the effectiveness of this treatment in reducing short-term and long-term OSA [11]. However, the patient did not need to expand the palate but due to his skeletal and occlusion characteristics an orthodontic appliance was chosen with the purpose of to solve sleep apnea on one side and be favorable to the resolution of its malocclusion on the other. The choice fell on a removable twin block to be worn afternoon and night for the first year and later only at night. As suggested by the literature, regardless of the presence of OSA it is important that the clinician uses these types of devices in pediatric patients only if a condition of retrognathic jaw is present. In fact the long-term results of these therapies are not yet certain and therefore it is important that the therapeutic objective is twofold: orthodontic and directed to solve the OSAS in the period of development [12]. The effects of this device were positive: on the one hand favored the correction of the dental class and on the other hand led to an $\mathrm{AHI}$ of 0.7 events per hour. The radiographic examination shows also which side effects have these devices: a proclination of the lower incisors and a retraction with uprighting of the upper incisors are visible (Fig. 7, Table 2). In line with recent studies in the literature there are no joint symptoms on the temporomandibular joint of the patient [13]. Obviously to obtain an excellent result also on an aesthetic level the therapy of choice still remains the orthognathic surgery but occlusaly the case can be terminated optimally even with only orthodontics (Fig. 6). The orthodontic evaluation therefore allows not only to choose the best treatment or jaw advancement device for the patient but also to identify the OSAS pathology early and send the patient to the appropriate specialist.

A multidisciplinary approach to OSAS pathology has become fundamental in both adult and pediatric patients. In the latter the role of orthodontic screening is even more important because of the large pool of young patients who visit every day. As we have seen, after solving the problems related to the otorhinolaryngology sphere an orthodontic intervention has given the possibility to reduce them even more by bringing the patient's sleep back to normal values. Treatment with MAD, supported by a correct medical history, diagnosis, and assessment of compliance is also valid in young patients and has to be associated with the resolution of other strictly orthodontic problems as demonstrated in this case. The knowledge of the diagnosis and therapy of these pathologies, together with the integration of specific standardized questionnaires included in his medical history, becomes therefore fundamental for the identification and treatment of this pathology. Finally, its role becomes essential when maxillofacial surgery or CPAP are excluded for reasons of age or will of the patient.

\section{Acknowledgments}

None. 


\section{Conflicts of Interest}

The authors have no financial conflicts of interest.

\section{Authors' Contribution}

Conceptualization: Bignotti D, De Stefani A, Mezzofranco L, Bruno G, Gracco A. Data curation: Bignotti D, De Stefani A, Mezzofranco L, Bruno G, Gracco A. Formal analysis: Bignotti D, De Stefani A, Mezzofranco L, Bruno G, Gracco A. Funding acquisition: Bignotti D, De Stefani A, Mezzofranco L, Bruno G, Gracco A. Investigation: Bignotti D, De Stefani A, Mezzofranco L, Bruno G, Gracco A. Methodology: Bignotti D, De Stefani A, Mezzofranco L, Bruno G, Gracco A. Project administration: Mezzofranco L, Gracco A. Resources: Bignotti D, De Stefani A, Bruno G. Software: Bignotti D, De Stefani A, Mezzofranco L, Bruno G, Gracco A. Supervision: Gracco A. Validation: De Stefani A, Mezzofranco L, Bruno G. Visualization: De Stefani A, Bruno G. Writing_original draft: Bignotti D. Writing_review \& editing: De Stefani A, Bruno G.

\section{REFERENCES}

1. Brunetti L, Rana S, Lospalluti ML, Pietrafesa A, Francavilla R, Fanelli $\mathrm{M}$, et al. Prevalence of obstructive sleep apnea syndrome in a cohort of 1,207 children of southern Italy. Chest 2001;120:1930-5.

2. Gislason T, Benediktsdóttir B. Snoring, apneic episodes, and nocturnal hypoxemia among children 6 months to 6 years old. An epidemiologic study of lower limit of prevalence. Chest 1995;107:963-6.

3. Marcus CL, Brooks LJ, Draper KA, Gozal D, Halbower AC, Jones J, et al. Diagnosis and management of childhood obstructive sleep apnea syndrome. Pediatrics 2012;130:576-84.

4. Joosten KF, Larramona H, Miano S, Van Waardenburg D, Kaditis AG, Vandenbussche N, et al. How do we recognize the child with OSAS? Pediatr Pulmonol 2017;52:260-71.
5. Capdevila OS, Kheirandish-Gozal L, Dayyat E, Gozal D. Pediatric obstructive sleep apnea: complications, management, and long-term outcomes. Proc Am Thorac Soc 2008;5:274-82.

6. Brietzke SE, Gallagher D. The effectiveness of tonsillectomy and adenoidectomy in the treatment of pediatric obstructive sleep apnea/hypopnea syndrome: a meta-analysis. Otolaryngol Head Neck Surg 2006; 134:979-84.

7. Gracco A, Bruno G, de Stefani A, Ragona RM, Mazzoleni S, Stellini E. Combined orthodontic and surgical treatment in a 8-years-old patient affected by severe obstructive sleep apnea: a case-report. J Clin Pediatr Dent 2018;42:79-84.

8. Marcus CL, Moore RH, Rosen CL, Giordani B, Garetz SL, Taylor HG, et al. A randomized trial of adenotonsillectomy for childhood sleep apnea. N Engl J Med 2013;368:2366-76.

9. Hawkins SM, Jensen EL, Simon SL, Friedman NR. Correlates of pediatric CPAP adherence. J Clin Sleep Med 2016;12:879-84.

10. Huynh NT, Desplats E, Almeida FR. Orthodontics treatments for managing obstructive sleep apnea syndrome in children: a systematic review and meta-analysis. Sleep Med Rev 2016;25:84-94.

11. Pirelli P, Saponara M, Guilleminault C. Rapid maxillary expansion (RME) for pediatric obstructive sleep apnea: a 12-year follow-up. Sleep Med 2015;16:933-5.

12. Behrents RG, Shelgikar AV, Conley RS, Flores-Mir C, Hans M, Levine M, et al. Obstructive sleep apnea and orthodontics: An American Association of Orthodontists White Paper. Am J Orthod Dentofacial Orthop 2019;156:13-28.

13. Crivellin G, Bruno G, De Stefani A, Mazzoli A, Mandolini M, Brunzini A, et al. Strength distribution on TMJ using mandibular advancement device for OSAS treatment : a finite element study. Dental Cadmos 2018;86:757-64 